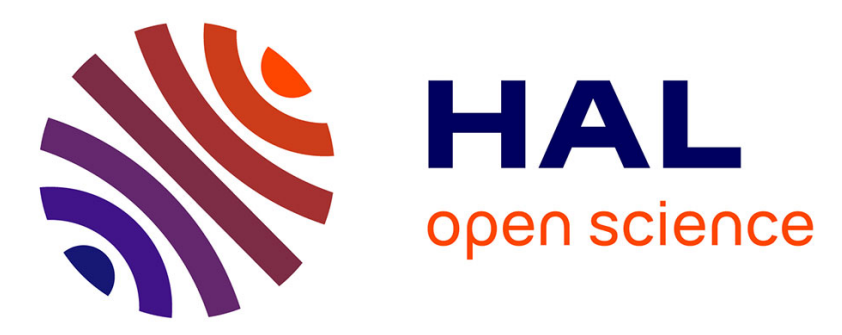

\title{
Proposition d'une méthode d'évaluation et de cartographie des risques liés aux lahars sur le volcan Merapi (Java, Indonésie)
}

Franck Lavigne, Jean-Claude Thouret

\section{- To cite this version:}

Franck Lavigne, Jean-Claude Thouret. Proposition d'une méthode d'évaluation et de cartographie des risques liés aux lahars sur le volcan Merapi (Java, Indonésie). Revue de Géographie Alpine / Journal of Alpine Research, 1994, 82 (4), pp.151-166. 10.3406/rga.1994.3781 . hal-02476172

\section{HAL Id: hal-02476172 \\ https://hal.science/hal-02476172}

Submitted on 3 Feb 2021

HAL is a multi-disciplinary open access archive for the deposit and dissemination of scientific research documents, whether they are published or not. The documents may come from teaching and research institutions in France or abroad, or from public or private research centers.
L'archive ouverte pluridisciplinaire HAL, est destinée au dépôt et à la diffusion de documents scientifiques de niveau recherche, publiés ou non, émanant des établissements d'enseignement et de recherche français ou étrangers, des laboratoires publics ou privés.

\section{(c)(1)}

Distributed under a Creative Commons Attribution| 4.0 International License 


\section{Proposition d'une méthode d'évaluation et de cartographie des} risques liés aux lahars sur le volcan Merapi (Java, Indonésie) Monsieur Franck Lavigne, M. Jean-Claude Thouret

\section{Citer ce document / Cite this document :}

Lavigne Franck, Thouret Jean-Claude. Proposition d'une méthode d'évaluation et de cartographie des risques liés aux lahars sur le volcan Merapi (Java, Indonésie). In: Revue de géographie alpine, tome 82, n4, 1994. pp. 151-166;

doi : https://doi.org/10.3406/rga.1994.3781

https://www.persee.fr/doc/rga_0035-1121_1994_num_82_4_3781

Fichier pdf généré le 22/04/2018 


\begin{abstract}
Abstract : A proposal for assessing and mapping lahar-related hazard zones on the volcano Mount Merapi (central Java, Indonesia). About 440000 people live at risk in areas prone to pyrodastic flows and lahars on the west and south flanks of the active stratovolcano Merapi. At least 23 major eruptions of the 61 reported eruptions since the mid-1500's (V.S.I., 1990) have triggered lahars which killed about 7000 people. The hazard-zone map of Merapi produced by the Volcanological Survey of Indonesia (1978) identifies three areas referred to as the forbidden zone, first danger zone and second danger zone, according to the different types of volcanic hazards. This map should now be revised, because its scale $(1 / 100000)$ lacks the detail necessary to accurately delimit the hazard zones, in particular valley channels likely to be swept by lahars. In addition, the map does not include social and economic factors which render people vulnerable and may lead to severe loss of life and property. Thus, a proposal is made for a combined appraisal and detailed mapping of hazard zones based on : (1) the characteristics, distribution, and sedimentology of recent deposits, and geometry of valley channels likely to be affected by lahars ; (2) social characteristics of people at risk
\end{abstract}

\title{
Résumé
}

Résumé : Sur les flancs du Merapi, strato-volcan actif de l'île de Java, plus de 440000 personnes vivent dans des zones menacées par des coulées pyroclastiques à blocs et cendres (nuées ardentes) et des lahars (coulées de débris et de boue). Sur les 61 éruptions du volcan recensées depuis 5 siècles (V.S.I., 1990), au moins 23 éruptions majeures ont été accompagnées de lahars, qui ont laissé un solde de 7000 victimes. Une carte des zones menacées par l'activité du Merapi (V.S.I., 1978), divise les flancs du volcan en trois zones, dites " interdites", de " premier » puis de " second danger ", en fonction de leur exposition aux différentes menaces volcaniques. Ce document devrait être révisé, car son échelle (1/100 000) ne permet pas de délimiter précisément les vallées menacées et il ne tient pas compte des différents facteurs de vulnérabilité des personnes et des biens. Nous proposons une méthode fondée sur un micro-zonage cartographique des secteurs affectés par les lahars (1/10 000), à partir de la distribution des dépôts récents, la sé- dimentologie des matériaux remobilisables et la morphométrie des chenaux des vallées empruntées par les écoulements. Le second volet de cette méthode est l'étude des facteurs de vulnérabilité de la population, des biens et de l'économie régionale. Menées conjointement, les deux recherches permettront de dresser une carte détaillée et intégrée des risques associés aux lahars, utili- sable par les responsables de la prévention des risques et de la gestion d'une crise volcanique au Merapi. 


\section{Proposition d'une méthode d'évaluation et de cartographie des risques liés aux lahars sur le volcan Merapi (Java, Indonésie)}

Résumé : Sur les flancs du Merapi, strato-volcan actif de l'île de Java, plus de 440000 personnes vivent dans des zones menacées par des coulées pyroclastiques à blocs et cendres (nuées ardentes) et des lahars (coulées de débris et de boue). Sur les 61 éruptions du volcan recensées depuis 5 siècles (V.S.I., 1990), au moins 23 éruptions majeures ont été accompagnées de lahars, qui ont laissé un solde de 7000 victimes. Une carte des zones menacées par l'activité du Merapi (V.S.I., 1978), divise les flancs du volcan en trois zones, dites " interdites", de " premier " puis de "second danger ", en fonction de leur exposition aux différentes menaces volcaniques. Ce document devrait être révisé, car son échelle $(1 / 100000)$ ne permet pas de délimiter précisément les vallées menacées et il ne tient pas compte des différents facteurs de vulnérabilité des personnes et des biens.

Nous proposons une méthode fondée sur un micro-zonage cartographique des secteurs affectés par les lahars $(1 / 10000)$, d̀ partir de la distribution des dépôts récents, la sédimentologie des matériaux remobilisables et la morphométrie des chenaux des vallées empruntées par les écoulements. Le second volet de cette méthode est l'étude des facteurs de vulnérabilité de la population, des biens et de l'économie régionale. Menées conjointement, les deux recherches permettront de dresser une carte détaillée et intégrée des risques associés aux lahars, utili- sable par les responsables de la prévention des risques et de la gestion d'une crise volcanique au Merapi.

Abstract : A proposal for assessing and mapping lahar-related hazard zones on the volcano Mount Merapi (central Java, Indonesia). About 440000 people live at risk in areas prone to pyroclastic flows and lahars on the west and south flanks of the active stratovolcano Merapi. At least 23 major eruptions of the 61 reported eruptions since the mid1500's (V.S.I., 1990) have triggered lahars which killed about 7000 people. The hazard-zone map of Merapi produced by the Volcanological Survey of Indonesia (1978) identifies three areas referred to as the forbidden zone, first danger zone and second danger zone, according to the different types of volcanic hazards. This map should now be revised, because its scale $(1 / 100000)$ lacks the detail necessary to accurately delimit the hazard zones, in particular valley channels likely to be swept by lahars. In addition, the map does not include social and economic factors which render people vulnerable and may lead to severe loss of life and property.

Thus, a proposal is made for a combined appraisal and detailed mapping of hazard zones based on : (1) the characteristics, distribution, and sedimentology of recent deposits, and geometry of valley channels likely to be affected by lahars ; (2) social characteristics of people at risk
Franck Lavigne* et Jean-Claude Thouret**1

Mots-clés : Indonésie, volcan Merapi, lahars, cartographie, prévention des risques, analyse de vulnérabilité.

Keywords : Indonesia, Merapi volcano, mapping, risk prevention, analysis of vulnerability

1. C.R.V.URA 10 CNRS * et CERAMAC* Université Blaise PascalClermont II, 29 Bd. Gergovia, 63000 Clermont-Ferrand. 
Illustration non autorisée à la diffusion.

1. Cicatrice probable de la caldera d'avalanche (Faille de Kukusan) et accidents cassants $\mathrm{N} 45$ et N135-165; 2. Collines de Gendol : dépôts d'avalanche de débris ; 3 . Dôme actuel et coulées de lave andésitique

historiques ; 4. Piémonts et vallées empruntés par les lahars ; 5 . Dépôts de cendres d'origine phréatomagmatique de Gumuk et de Simbasari ; 6. Ellipses des retombées attendues (isoplète $1,6 \mathrm{~cm}$ ) pour une colonne éruptive de $14 \mathrm{~km}$ de hauteur. and assessment of potential loss of property, cultivated land, and economic goods at risk. The aim of such an approach is to provide a better assessment of lahar-related hazard zones which can be used by decision makers in the event of increasing eruptive activity and crisis on Mount Merapi.

\section{Introduction}

Sur les flancs du Merapi (7³3'S-1 10²7'E, $2965 \mathrm{~m}$ ), le volcan le plus actif et dangereux de l'Indonésie (d'après les critères volcanologiques et démographiques, in Crandell et al., 1984), vivent 1300000 personnes. La densité moyenne, de 1500 habitants au $\mathrm{km}^{2}$, s'avère extrêmement élevée, d'autant plus que le cône sommital (au-dessus de $1500 \mathrm{~m}$ ) n'est pas habité en permanence. Environ $\mathbf{4 4 0} 000$ personnes habitent dans les zones menacées par des écoulements pyroclastiques ou des lahars (coulées de débris et de boue, concentration en sédiments $\geq 60 \%$ en volume). A titre d'exemple, la dernière éruption modérée à " nuées ardentes " du 22 novembre 1994 s'est soldée par 63 victimes et des dommages évalués à 3,5 millions de francs (F. Lavigne et V.S.I., com. pers.)

Trois types de lahars sont distingués selon leur mode de déclenchement et leur source en eau (Neall, 1976 ; Lavigne, 1993) : lahars primaires issus de la liquéfaction d'écoulements pyroclastiques, lahars générés par la vidange d'un lac et lahars secondaires pluviaux. Tous s'écoulent le long des vallées jusqu'à 20-30 km du dôme sommital (Fig. 1) et ils se produisent aussi pendant les phases de repos du volcan, à la suite de la remobilisation des matériaux pyroclastiques par de fortes pluies. L'évaluation de cette menace demeurant difficile (Tabl. I), nous proposons une méthode fondée sur le micro-zonage cartographique dans deux secteurs-pilotes, associé à une étude de vulnérabilité des personnes et des biens dans les zones exposées (Tabl. II).

\section{Evaluation de la menace et zonage traditionnel autour du Merapi}

L'évaluation de la menace passe par une analyse de l'extension, du volume et de la récurrence des lahars historiques. Nous préférons le terme de menace ou phénomène naturel menaçant à 
celui d'aléa, dont l'origine étymologique ne convient pas à l'acception communément admise du risque naturel (= phénomène naturel créateur de dommage $x$ endommagement potentiel des biens et des personnes).

\section{Extension, récurrence des lahars historiques et dommages engendrés}

Du fait de l'abondance de matériaux mobilisables sur les flancs ouest et SW du Merapi, les lahars constituent la principale menace le long des 12 principales rivières radiales qui débouchent 


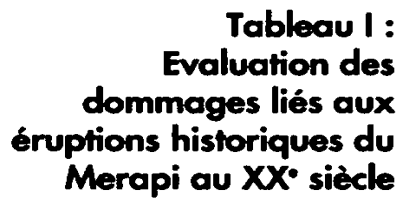

2. Depuis 5 siècles, 23 des 01 éruptions recensées N.S.I., 1990) auraient déclenché des lahars, qui ont provoqué des pertes humaines et matérielles considérables lors des éruptions majeures en $1849,1871-73$ et 1930

31 (1370 morts). Des lahars meurtriers ont également été déclenchés lors d'éruptions modérées en 1672 (3000 morts). 1822-23, 1832-35, 1902-1904, 1920 , $1953-54$ et 1976.

\begin{tabular}{|c|c|c|c|}
\hline $\begin{array}{l}\text { Crise volcanique } \\
\text { au XX· Siècle }\end{array}$ & $\begin{array}{c}\text { Pertes humaines } \\
\text { EP écoulements } \\
\text { pyroclastiques - } \\
\text { L lahars }\end{array}$ & $\begin{array}{l}\text { Nombre } \\
\text { de villages } \\
\text { affectés }\end{array}$ & $\begin{array}{l}\text { Nombre } \\
\text { de Lahars } \\
\text { primaires } \\
\text { recensés }\end{array}$ \\
\hline $1902-04$ & 16 (EP) & 3 & - \\
\hline $1920-21$ & 35 (EP) & 1 & $3(12 / 10 / 1920)$ \\
\hline 1922 & - & - & - \\
\hline $1930-31$ & $1369(E P+L)$ & 42 & $\begin{array}{l}\text { plusieurs dizaines } \\
19 / 12 / 30,2 / 1 \\
\& 27 / 41931\end{array}$ \\
\hline février 1932 & - & - & 5 (17 février) \\
\hline $1942-43$ & - & - & - \\
\hline $1953-54$ & 64 (EP) & 6 & - \\
\hline $1955-58$ & - & - & - \\
\hline 1961 & $8(E P+L)$ & 10 & 3 (27-28 novembre) \\
\hline $1967-68$ & - & - & - \\
\hline janvier 1969 & $3(E P+L)$ & 26 & 4 (7-8 janvier) \\
\hline $1973-75$ & 9 (L) & plusieurs dizaines & - \\
\hline $\begin{array}{l}\text { novembre- } \\
\text { décembre } 1976\end{array}$ & $29(L)$ & plusieurs dizaines & 2 (25 novembre) \\
\hline janvier 1979 & - & - & - \\
\hline juin 1984 & - & - & - \\
\hline février 1992 & - & - & - \\
\hline $\begin{array}{l}22 \text { novembre - } \\
7 \text { décembre } 1994\end{array}$ & 63 (EP) & 4 & - \\
\hline
\end{tabular}

sur le piémont ouest et dans la plaine de Yogjakarta vers le Sud (Fig. $1 \& 2$ ). Au Merapi, les lahars sont déclenchés par deux processus : (1) les lahars primaires proviennent de la liquéfaction d'écoulements pyroclastiques canalisés ; (2) les lahars pluviaux (chauds et synéruptifs ou froids et post-éruptifs) surviennent lorsque le seuil pluviométrique atteind $40 \mathrm{~mm} / \mathrm{h}$ durant au moins 2 heures lors de la mousson, de novembre à mars ${ }^{2}$. Malgré l'état fragmentaire des informations disponibles, les dégâts causés par les 17 éruptions du XX' siècle ont été estimés (Tabl. I ; Simkin et al., 1981 ; V.S.I., 1990). Au XX' siècle, plus de 1500 personnes ont trouvé la mort, une centaine de villages ont été entièrement ou partiellement détruits, près de 2800 maisons ont été rasées et les terres cultivées perdues se comptent en milliers d'hectares. La récurrence des lahars est de 10 à 30 ans pour les écoulements majeurs et de 4 à 6 ans pour les secondaires $\left(\leq 1 \times 10^{6} \mathrm{~m}^{3}\right.$ ). Le volume approximatif des futurs lahars et les cotes d'écoulement en un point donné 


\begin{tabular}{|c|c|c|c|}
\hline $\begin{array}{l}\text { Géologie et } \\
\text { géomorphologie } \\
\text { des vallées et des dépôts } \\
\text { de lahars }\end{array}$ & $\begin{array}{l}\text { Distribution des dépôts } \\
\text { pyroclastiques et } \\
\text { lahariques récents }\end{array}$ & $\begin{array}{l}\text { Objectifs poursuivis } \\
\text { Déterminer les vallées et } \\
\text { chenaux les plus } \\
\text { empruntés par les lahars } \\
\text { récents } \\
\text { Repérer et calculer le } \\
\text { volume des dépôts des } \\
\text { coulées pyroclastiques } \\
\text { de } 1984,1992 \text { et } 1994 \\
\text { pour évaluer la } \\
\text { trajectoire des futurs } \\
\text { lahars pluviaux }\end{array}$ & $\begin{array}{l}\text { Outils et réalisations } \\
\text { Cartes des dépôts } \\
\text { pyroclastiques récents et } \\
\text { des lahars au } 1 / 10000 \\
\text { Exégèse des textes } \\
\text { historiques et des } \\
\text { cartes existantes } \\
\text { Photo-interprétation } \\
\text { et télédétection } \\
\text { (images SPOT } 2 \text { et } \\
\text { radar) }\end{array}$ \\
\hline $\begin{array}{l}\text { Morphométrie des } \\
\text { vallées } \\
\text { exposées }\end{array}$ & $\begin{array}{l}\text { Etude morphologique et } \\
\text { morphométrique } \\
\text { détaillée des vallées } \\
\text { menacées (Putih, } \\
\text { Krasak, Bojong, Kuning, } \\
\text { Gendol) }\end{array}$ & $\begin{array}{l}\text { Disposer de données } \\
\text { géométriques sur les } \\
\text { chenaux (largeur, pente, } \\
\text { hauteur des berges) } \\
\text { avant le passage des } \\
\text { prochains lahars }\end{array}$ & $\begin{array}{l}\text { - Profils longitudinaux } \\
\text { - Profils transversaux } \\
\text { réitérés dans une ou } \\
\text { deux vallées menacées }\end{array}$ \\
\hline $\begin{array}{l}\text { Sédimentologie et } \\
\text { études géotechniques } \\
\text { des matériaux } \\
\text { mobilisables }\end{array}$ & $\begin{array}{l}\text { Etude physique } \\
\text { et sédimentologique } \\
\text { des matériaux } \\
\text { remobilisables }\end{array}$ & $\begin{array}{l}\text { - Evaluer le volume } \\
\text { de matériaux } \\
\text { remobilisables dans les } \\
\text { vallées } \\
\text { - Analyser les caractères } \\
\text { physico-hydriques des } \\
\text { téphras : épaisseur, } \\
\text { granulométrie, cohésion, } \\
\text { densité, teneur en eau et } \\
\text { vitesse d'infiltration }\end{array}$ & $\begin{array}{l}\text { Extension, géométrie et } \\
\text { caractéristiques } \\
\text { lithologiques des } \\
\text { matériaux } \\
\text { remobilisables } \\
\text { Analyses } \\
\text { sédimentologiques et } \\
\text { géotechniques } \\
\text { Profils réitérés et } \\
\text { morphométrie } \\
\text { des chenaux empruntés }\end{array}$ \\
\hline
\end{tabular}

peuvent être évalués par la corrélation de la distribution de leurs dépôts récents (à partir d'une image SPOT 2 XS, Fig. 2) avec trois magnitudes d'éruptions survenues dans le passé : éruption majeure, Indice d'Explosivité Volcanique VEI > 3, volume de téphras $4 \times 10^{7} \mathrm{~m}^{3}$, comme en 1930-31 ; éruption modérée VEI 2-3, $1-4 \times 10^{7} \mathrm{~m}^{3}$, comme en $1953-54$ et 1961 ; éruption mineure VEI 2, 1-4 x 106 $\mathrm{m}^{3}$, comme en 1969, 1984, 1992 et 1994 (V.S.I., 1989, 1990 et com. pers. ; Simkin et al., 1981 ; GVN, 1994).

A la différence des lahars du Kelud (Java est), générés par la vidange synéruptive d'un lac de cratère, les lahars du Merapi ont une moindre proportion d'eau ( $\leq 20 \%$ en poids), une vitesse
Tableau II

Méthode d'évaluation des risques liés aux lahars du Merapi.

A-Micro-zonage cartographique des zones exposées aux écoulements 


\begin{tabular}{|c|c|c|c|}
\hline Population vulnérable & $\begin{array}{l}\text { - Répartition et densité } \\
\text { de la population dans } \\
\text { les zones menacées, } \\
\text { notamment les vallées } \\
\text { - Evolution } \\
\text { démographique } \\
\text { et prévisions } \\
\text { socio-démographiques }\end{array}$ & $\begin{array}{l}\text { - Déterminer les facteurs } \\
\text { socio-démographiques } \\
\text { de la vulnérabilité de la } \\
\text { population selon divers } \\
\text { scénarios } \\
\text { - Evaluer le nombre } \\
\text { potentiel de victimes, de } \\
\text { blessés et de sans-abris } \\
\text { potentiels }\end{array}$ & $\begin{array}{l}\text { - Cartes des densités de } \\
\text { population au } \\
1 / 10000 \\
\text { - Documents et cartes de } \\
\text { l'évolution récente et de } \\
\text { la dynamique de la } \\
\text { population } \\
\text { (migrations) }\end{array}$ \\
\hline $\begin{array}{l}\text { Biens mobiliers ef } \\
\text { immobiliers vulnérables }\end{array}$ & $\begin{array}{l}\text { - Site et localisation } \\
\text { - Architecture et } \\
\text { matériaux de } \\
\text { construction }\end{array}$ & $\begin{array}{l}\text { - Déterminer le type et le } \\
\text { nombre des bâtiments } \\
\text { d'habitation, scolaires, } \\
\text { sanitaires, religieux et } \\
\text { artisanaux } \\
\text { - Localiser et évaluer le } \\
\text { nombre de bâtiments } \\
\text { capables de résister au } \\
\text { passage d'un lahar } \\
\text { - Estimer le coût des } \\
\text { pertes éventuelles }\end{array}$ & $\begin{array}{l}\text { - Cartes de densité et } \\
\text { évolution de l'habitat } \\
\text { dans les zones } \\
\text { menacées } \\
\text { - Cartes de la qualité du } \\
\text { bâti et du type de } \\
\text { constructions } \\
\text { hétérogènes } \\
\text { - Estimation du coût } \\
\text { direct (réparation) et } \\
\text { indirect (remplacement) } \\
\text { des bâtiments }\end{array}$ \\
\hline $\begin{array}{l}\text { Infrastructures et } \\
\text { activités économiques } \\
\text { vulnérables }\end{array}$ & $\begin{array}{l}\text { - Infrastructures, } \\
\text { aménagements, } \\
\text { équipements, réseaux de } \\
\text { communications et } \\
\mathrm{d}^{\prime} \text { informations } \\
\text { - Terres agricoles et } \\
\text { forêts } \\
\text { - Entrepôts et bâtiments } \\
\text { à vocation agro- } \\
\text { alimentaire et artisanale }\end{array}$ & $\begin{array}{l}\text { - Recenser les } \\
\text { infrastructures menacées } \\
\text { par les lahars } \\
\text { - Evaluer la Surface } \\
\text { Agricole Utilisable } \\
\text { menacée et la valeur des } \\
\text { différentes productions } \\
\text { - Estimer le coût des } \\
\text { dégâts éventuels }\end{array}$ & $\begin{array}{l}\text { - Carte des } \\
\text { infrastructures avec } \\
\text { hiérarchisation selon le } \\
\text { niveau d'exposition aux } \\
\text { lahars } \\
\text { - Cartes intégrées ef } \\
\text { évolutives de type S.I.G. } \\
\text { - Cartographie de } \\
\text { la SAU menacée, selon } \\
\text { la valeur des } \\
\text { productions }\end{array}$ \\
\hline $\begin{array}{l}\text { Objectif final de } \\
\text { prévention }\end{array}$ & $\begin{array}{l}\text { Evaluer les scénarios } \\
\text { éruptifs possibles pour } \\
\text { élaborer des plans de } \\
\text { contingence et } \\
\text { d'évacuation }\end{array}$ & $\begin{array}{l}\text { Repérer l'accès aux } \\
\text { zones de refuge et de } \\
\text { secours selon divers } \\
\text { scénarios pour déceler } \\
\text { les blocages éventuels }\end{array}$ & $\begin{array}{l}\text { Elaborer des plans } \\
\text { d'évacuation précis et } \\
\text { de secours en cas de } \\
\text { catastrophes selon les } \\
\text { scénarios }\end{array}$ \\
\hline
\end{tabular}

Tableau II B (quelques $\mathrm{m} / \mathrm{s}$ ) et un débit (maximum $825 \mathrm{~m}^{3} / \mathrm{s}$ ) plus faibles et ils se déposent plus près du sommet (Lavigne \& Thouret, des personnes et des biens dans les vallées menacées 1994). En conséquence, les caractéristiques morphométriques englobent une surface recouverte de quelques centaines d'hec- 

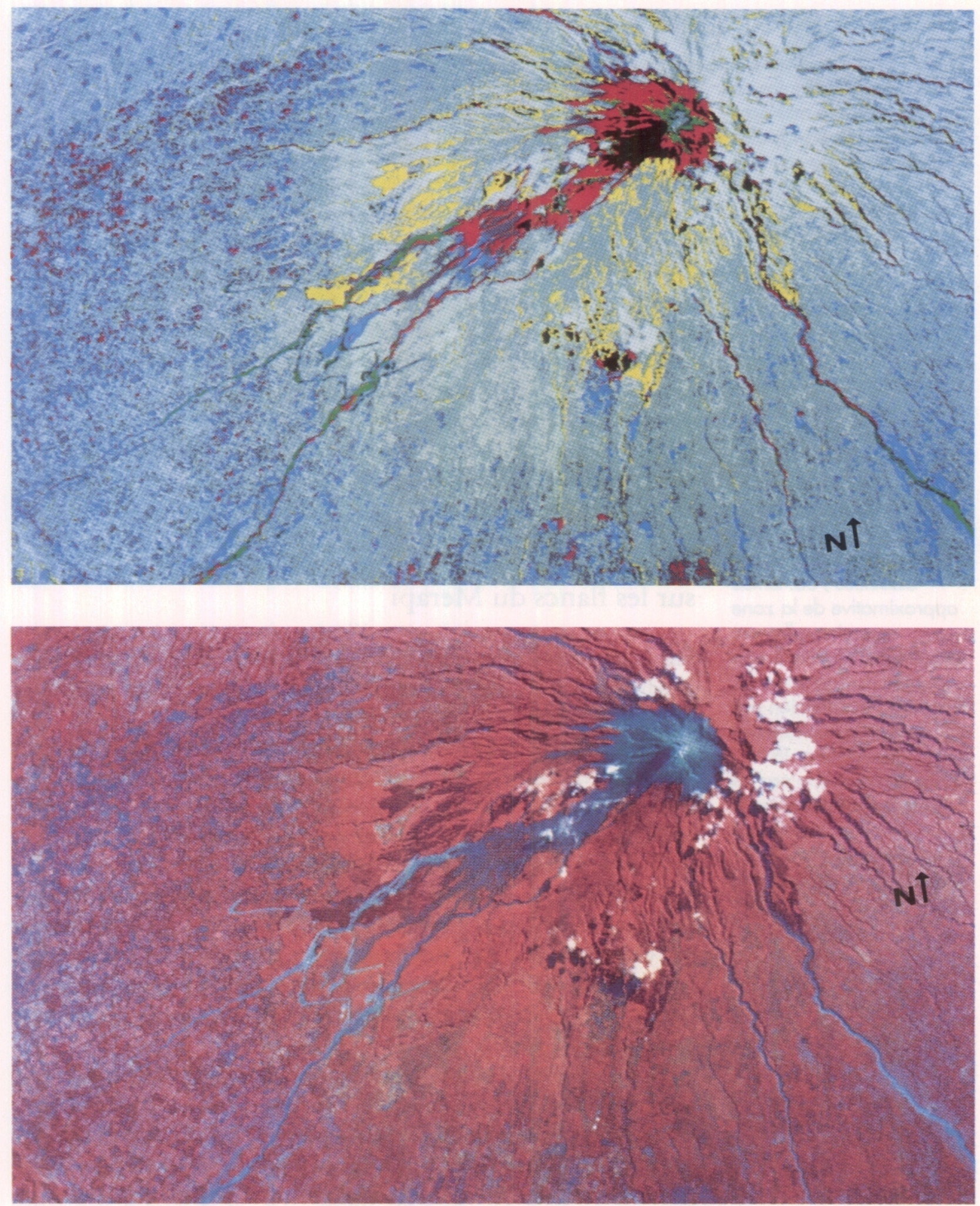

Figure 2

A. Image SPOT 2 XS (9/08/1990) centrée sur le Merapi, canal infra-rouge (échelle $1 / 80000$ environ). B. Distribution de certains produits pyroclastiques et des dépôts de lahars autour du Merapi, repérés sur une image SPOT 2 XS (9/08/1990) et classés par traitement statistique (logiciel Planète).

Couleur rouge $=$ dépôts de nuées ardentes; orange $=$ dépôts de nuées remaniées $;$ vert $=$ dépôts de lahars récents ; bleu marine $=$ dépôts de lahars plus anciens ; jaune $=$ cendres épaisses. 
Figure 3

Carte préliminaire de zonage des menaces volcaniques principales autour du strato-cône du Merapi (révision des documents du V.S.I., 1978, 1989).

1. Dôme actuel (andésite); 2. Flanc instable du dôme et talus de blocs écroulés ;

3. Ecoulements pyroclastiques à blocs ef cendres fréquents ;

4. Ecoulements

pyroclastiques potentiels

(《 nuées ardentes") ;

5a. Limite probable des explosions latéralement dirigées à déferlantes associées ; 5b. Limite approximative de la zone exceptionnellement affectée par une explosion dirigée du type Mont St.

Helens; 6. Direction des écoulements pyroclastiques de 1992 et 1994 ;

7. Zone recouverte par les produits de déferlantes ef par les coulées pyroclastiques du 22 novembre au 7 décembre 1994. tares par vallée, une longueur généralement inférieure à $20 \mathrm{~km}$ et une épaisseur de 0,5 à $5 \mathrm{~m}$. Leur faciès non compacté contient des blocs denses en vrac (issus des " nuées ardentes"), hétérométriques et anguleux jusqu'à grande distance, dans une matrice sablo-graveleuse, très pauvre en argile. En revanche, à l'instar de ceux du Kelud, les lahars du Merapi (grossiers, à clastes prédominants, avec une concentration en sédiments $\mathrm{Cv} \geq 60 \%$ en volume) se transforment rapidement en écoulements hyperconcentrés ( $\mathrm{Cv}$ 40-60\%) et au-delà en écoulements torrentiels ( $\mathrm{Cv} \leq 40 \%)$. Ces écoulements de transition engendrent des inondations par débordements fréquents sur les pentes faibles des cônes de déjection qui forment les piémonts ouest et sud du Merapi. Ces écoulements provoquent donc des dégâts considérables à une distance bien supérieure à celle des lahars grossiers, davantage chenalisés dans les gorges radiales à l'amont du strato-volcan.

\section{Le zonage actuel des menaces volcaniques sur les flancs du Merapi}

Sur la carte des zones menacées par l'activité du Merapi (V.S.I.Pardyanto et al., 1978, 1/100 000), le volcan et ses piémonts sont divisés en trois secteurs (Fig. $1 \& 3$ ).

- (1) la zone interdite, correspondant à la partie supérieure de l'édifice volcanique, est fréquemment affectée par des écoulements pyroclastiques et des projections de téphras, y compris balistiques. Alors que ce secteur devrait être abandonné en permanence, il rassemblait en 1990 plus de 50000 personnes.

- (2) la zone de premier danger regroupe les régions pouvant être menacées lors d'éruptions violentes (pliniennes, péléennes ou vulcaniennes) par des retombées ou par des lahars. Elles sont censées être hors d'atteinte des écoulements pyroclastiques et des coulées de lave. Au moins 86000 personnes peuplaient cette zone en 1990.

- (3) la zone de second danger correspond aux lits majeurs et aux rives des cours d'eau radiaux drainant les flancs du volcan, surtout vers l'Ouest et le Sud. Les manifestations des lahars, des écoulements torrentiels et des crues peuvent les affecter, y compris entre 20 et $30 \mathrm{~km}$ du sommet. La population installée dans cette zone dépassait 110000 personnes en 1990. Il faut doubler ce chiffre si l'on considère la population potentiellement affectée par les retombées de lapilli et de cendres à partir de panaches éruptifs élevés, dans un rayon de 6 à $15 \mathrm{~km}$ vers le Nord, l'Ouest et le Sud (Fig. 1). 


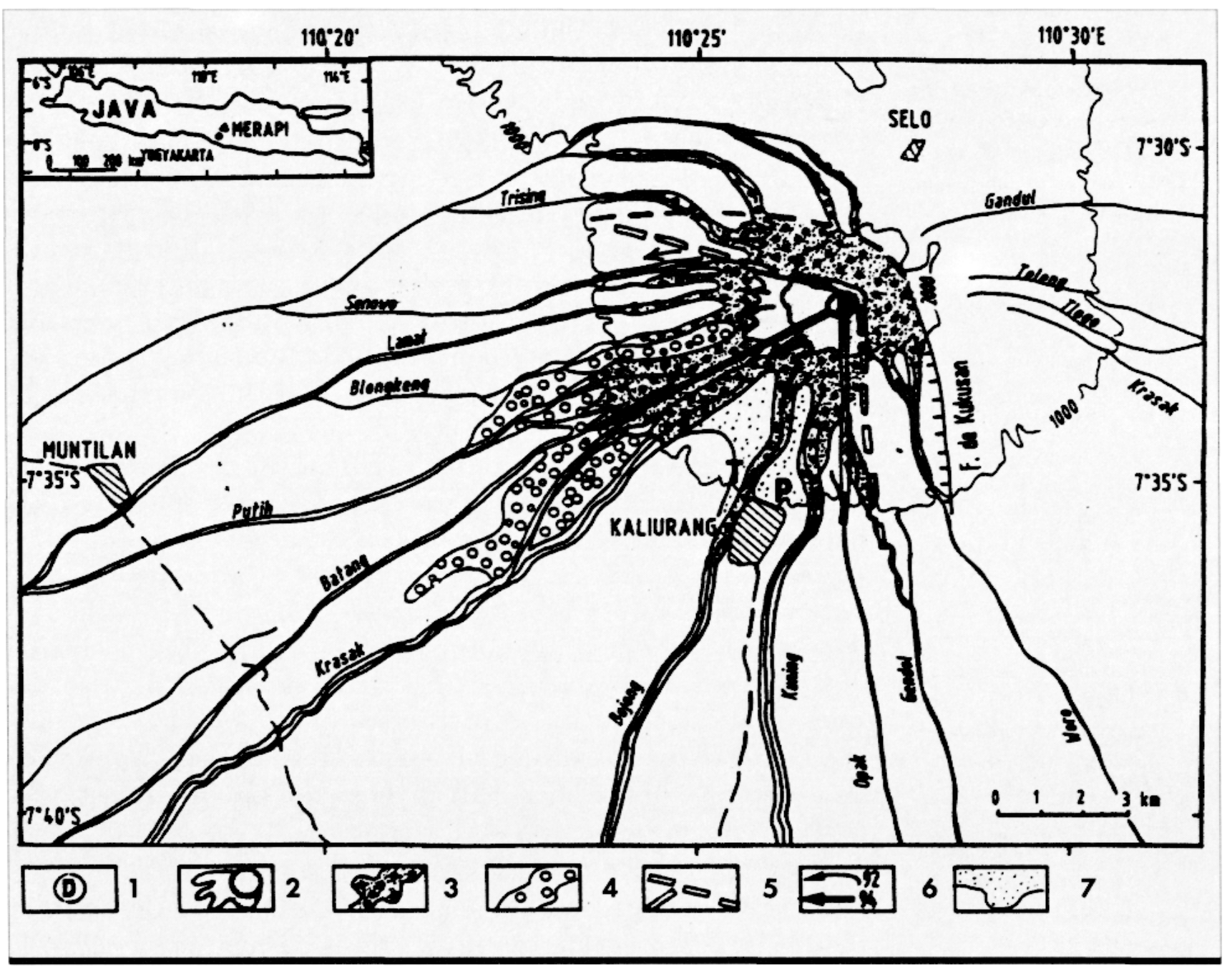

Le taux de croissance variable de la population et les migrations internes ou externes ne permettent pas d'évaluer avec certitude les effectifs directement menacés. Cependant, sur le secteur SSW du volcan affecté par l'éruption du 22 novembre 1994, dans un rayon de 4 à $12 \mathrm{~km}$ autour du dôme sommital, F. Lavigne a comptabilisé environ 68500 personnes : 15400 dans la zone interdite, 13500 dans celle de premier danger et 39600 dans celle de second danger.

3. Ce zonage traditionnel devra être révisé pour plusieurs raisons

- (1) La carte élaborée pour le Merapi, comme les autres cartes de menaces en Indonésie, suit une approche géologique : elle définit les zones d'extension potentielle de plusieurs types de menaces en fonction de la nature des éruptions reconnues dans le passé et de la distribution de leurs produits (surtout ceux de 
l'éruption de 1930-31). Ce type de carte, fréquemment utilisé, représente uniquement les limites extrêmes supposées pour le champ d'action de chaque menace, sans calcul statistique de la récurrence (cf. $\$ 1$. , ci-dessus).

On considère que les éruptions explosives seront dirigées verticalement, mais des explosions latéralement dirigées et des écoulements pyroclastiques (déferlantes) correspondant à des effondrements partiels de dômes en construction ont couramment lieu au Merapi : elles ont pour conséquence d'influencer la trajectoire et la dynamique des lahars qui leur ont succédé.

- (2) La préparation traditionnelle d'une carte des menaces volcaniques en Indonésie repose sur plusieurs présupposés (Kusumadinata in Crandell, 1984) qu'il convient de réviser :

- Les futures éruptions se produiraient dans le cratère principal ou dans un autre cratère qui a été actif dans le passé. La possibilité d'ouverture de nouveaux évents n'est donc pas prise en considération. Or, de fréquents changements de points de sortie se sont produits récemment au Merapi (1942-43, 1967, 1984, $1992,1994)$ et le dôme actuel en croissance quasi-continue depuis 1989 a d'abord recouvert le dôme précédent vers le NW en 1990-92, puis vers le SW depuis l'été $1994^{3}$.

- la morphologie du volcan ne changerait pas de manière appréciable entre la date de préparation de la carte et celle de la prochaine éruption. Or, la morphologie des chenaux susceptibles d'être empruntés par des lahars a subi de fortes modifications depuis 1978, date de parution de la carte (par exemple dans la Kali Putih : Sumaryono, 1992). Les nuées ardentes qui s'écoulaient vers le $S W$ depuis une trentaine d'années (à partir des dômes croissant dans le cratère de 1961 égueulé dans cette direction) ont pris une nouvelle direction vers l'Ouest en 1992,

3. Ce dôme instable, dont le volume est estimé à $10-11 \times 10 \mathrm{~m}$ : (Kelfoun et al., 1994, 19951, a menacé le flanc WNW Inuées ardentes de février 1992, Kali [=rivière] Senowo et Sat), puis le flanc SW (nuées ardentes de novembre 1994, Kali

Bojong et Krasakl et menace actuellement le flanc sud (Kali Gendol et Kuning: Fig. 1 \& 31. du fait de la croissance du dôme actuel vers l'WNW. Depuis 1994, ce dôme croît vers le SSW en débordant la paroi sud du cratère et des écoulements pyroclastiques ont été observés dans cette direction (22 novembre-7 décembre 1994 ; GVN, 1994 et Fig. 3). Ils sont maintenant attendus sur le flanc sud, déjà fragilisé par la fracturation et l'altération hydrothermale (bassin amont de la Kali Gendol). La déstabilisation du flanc sud fournirait des matériaux volumineux pour les lahars potentiels. Des vallées peu parcourues par les lahars depuis plus de 30 ans risquent d'être empruntées dans un avenir proche, comme celles qui, drainant le flanc sud, débouchent dans la plaine de Yogyakarta (ville de 500000 habitants en 1990 ; Fig. 1 \& 2). 
- (3) Comme la plupart des cartes existantes, l'échelle adoptée $(1 / 100000)$ est trop petite pour permettre un véritable zonage des menaces et des risques volcaniques en général et des lahars en particulier. Il est préférable de dresser une carte de menaces, puis une carte de risques à grande échelle ( $1 / 5000$ à $\left.1 / 2000^{\circ}\right)$ dans les zones urbanisées très exposées, à l'instar de la Kali Code (Thouret, 1990 ; Thouret \& Laforge, 1994).

\section{Méthodologie pour le micro-zonage et l'évaluation de la vulnérabilité dans les zones exposées aux lahars}

\section{Le micro-zonage cartographique}

Le micro-zonage des lahars s'appuiera sur les analyses de récurrence effectuées, sur les estimations des dommages recensés au cours des dernières éruptions étudiées et sur la distinction concernant la dynamique d'écoulement et ses effets, opérée entre les lahars primaires et les lahars secondaires ou pluviaux.

Le tableau II-A présente les grandes étapes de l'élaboration d'un micro-zonage cartographique des lahars au Merapi. L'objectif de la méthode est de disposer de données quantitatives précises (volume de matériaux pyroclastiques remobilisables dans les vallées, données morphométriques des chenaux susceptibles d'être parcourus, etc.), directement applicables à l'élaboration de scénarios. Ceux-ci pourront s'appuyer sur un Modèle Numérique de Terrain précis (C.R.V., Université Blaise Pascal : Kelfoun, 1994), créé par ISTAR à partir d'un couple d'images SPOT 2 Panchromatiques (résolution $10 \mathrm{~m}$ ), et sur une ortho-image, obtenue par le couplage d'une scène SPOT 2 P du 15/06/1987 et d'un M.N.T. La précision de ce dernier document permettra de localiser les lieux où les débordements et les défluviations sont possibles. Ces phénomènes fréquents sont dus à la faible pente des cônes de déjection volcano-détritiques formés au pied du strato-cône et à la faible profondeur des chenaux et des berges, dont les cotes altimétriques sont fréquemment dépassées par les écoulements habituels des lahars.

Dans le cas des lahars pluviaux, les plus fréquents, l'analyse porte sur quatre paramètres caractérisant les pluies capables de mobiliser les matériaux et de déclencher leur écoulement : l'intensité pluviométrique enregistrée, la durée de la pluie de l'évé- 
nement déclencheur, la précipitation totale de l'événement et la précipitation la plus intense enregistrée durant 10 minutes d'une forte averse. A l'heure actuelle, un seuil d'intensité pluviométrique unique de $40 \mathrm{~mm}$ de pluies tombées en $2 \mathrm{~h}$ sert de référence au V.S.I. pour prévoir le déclenchement d'un lahar. Or, un tel seuil varie en fonction d'autres facteurs comme la durée de l'averse et les paramètres morpho-sédimentologiques (Tabl. II-A). Quelques seuils d'intensité pluviométrique devront faire l'objet de calculs statistiques, en fonction de l'altitude, sur les pentes du Merapi.

\section{Evaluation de la vulnérabilité dans les zones menacées par les lahars}

La seconde phase dans l'évaluation des risques consiste à étudier les différents facteurs de vulnérabilité à l'intérieur des zones menacées. Trois groupes de facteurs seront approchés au moyen d'enquêtes (Tabl. II-B).

- (1) Les facteurs socio-démographiques de la vulnérabilité des populations

La densité de la population dans les zones menacées par les lahars constitue le principal facteur quantitatif de la vulnérabilité. La première démarche consiste à collecter des données démographiques, surtout le nombre d'habitants de chaque commune (desa), et si possible des villages et des hameaux (dusun). En Indonésie, cette étape préliminaire s'avère particulièrement longue et difficile (Lavigne, 1993), car les statistiques concernant les dusun ne sont pas toujours centralisées au niveau du desa. Cette banque de données rend ensuite possible l'élaboration d'une carte de répartition de la population au 1/10 000. La superposition de cette carte avec celle des zones menacées permettra ainsi d'évaluer le nombre de sinistrés potentiels lors du passage du prochain lahar important. Une telle carte devra être régulièrement remise à jour, compte-tenu des migrations et de la forte croissance démographique sur les flancs du volcan (environ $0,5 \%$ par an dans la zone la plus exposée aux lahars).

- (2) Les facteurs techniques et fonctionnels de la vulnérabilité des biens

Une étude détaillée du type d'habitat, de la qualité du bâti et des matériaux de construction dans les zones exposées aux lahars devra compléter les précédentes investigations. La qualité du 
bâti pourra être évaluée de manière précise par une cartographie de détail (1/5000 à 1/2000 dans la Kali Code, à proximité de Yogyakarta) et par des enquêtes portant sur les maisons d'habitation, les entrepôts et les bâtiments scolaires, religieux, hospitaliers ou à vocation industrielle. Le tableau II-B rappelle les opérations et les thèmes de cette investigation, les techniques employées et les réalisations attendues. A cet inventaire technique s'ajoutera un recensement des organismes et des moyens mis en oeuvre pour les secours en cas de crise et de catastrophe (postes de secours, personnel, bâtiments, réseaux de communications et d'énergie, flux d'informations et d'alerte, etc).

\section{- (3) Les facteurs de vulnérabilité économique}

La troisième étape de la méthode proposée consiste en une évaluation de la vulnérabilité des infrastructures, équipements et moyens de production dans la zone menacée : routes, chemins de fer, barrages, industries, commerces et terres agricoles. Cette évaluation a un double objectif : (1) estimer le coût des pertes éventuelles et leurs conséquences directes et indirectes sur l'économie régionale ; (2) repérer l'accès aux zones de refuge et aux secours selon divers scénarios pour déceler les blocages éventuels en cas de crise et de catastrophe. Plusieurs documents peuvent être ainsi réalisés à partir du cadastre, de photos aériennes et d'enquêtes : carte des terres cultivables menacées selon la valeur des productions, infrastructures endommageables classées selon l'impact des dommages potentiels et leurs conséquences socio-économiques, etc.

L'ultime étape de cette méthode d'évaluation des risques sera la superposition de tous les documents précédents, pour une corrélation spatiale sur un support cartographique évolutif de type S.I.G., appuyé sur un M.N.T. Nous envisageons de réaliser un document détaillé sur deux secteurs-pilotes choisis dans la région peuplée et affectée depuis la fin de 1994 : vallée de la Kali Bojong, à proximité de la ville de Kaliurang et en aval, vallée de la Kali Code, dans le périmètre urbain de Yogyakarta.

\section{Conclusion : intérêts et difficultés de la méthode proposée}

A l'heure actuelle, la plupart des cartes dites de risques autour des volcans actifs comportent deux défauts : leur échelle est trop petite, comme au Merapi (1/100 000) et elles ne représen- 
tent le plus souvent que les phénomènes volcaniques menaçants. Ce genre de document doit évoluer vers une carte de risque intégrée, rassemblant les données géologiques classiques, les calculs statistiques de récurrence (Newhall, 1984), mais aussi les facteurs socio-démographiques, économiques et techniques de la vulnérabilité dans les zones menacées (D'Ercole, 1991 ; Thouret \& D'Ercole, 1995).

L'intérêt majeur de cette méthode d'évaluation des risques est l'élaboration d'un document intégré à grande échelle $(1 / 10000$ à $1 / 2000)$ et évolutif. Ce document préparé en 1994-95 pourra être directement utilisable par les scientifiques, mais aussi par les autorités responsables de la prévention des risques et de l'évacuation en cas d'alerte. Néanmoins, cette méthode se heurte à des difficultés, à l'amont comme à l'aval des investigations.

A l'amont, l'évaluation des risques associés aux coulées pyroclastiques et aux lahars requiert une très bonne connaissance des mécanismes de déclenchement et de la dynamique de ces écoulements. Au Merapi, le volcan le plus étudié de Java (Berthommier, 1990, Berthommier et al., 1992, Vincent, 1992), on ne disposait que de rapports descriptifs de quelques lahars. Or, ces 10 dernières années ont vu s'accomplir des progrès remarquables dans ce domaine d'étude grâce aux travaux du Sabo Technical Center (Yogyakarta), un organisme transposant en Indonésie les techniques de surveillance japonaises les plus sophistiquées (S.T.C., 1992) : capteurs de précipitations mesurant l'intensité des pluies en $1 \mathrm{~h}, 30$ et 10 minutes, limnimètres dans les rivières, détecteurs de lahars par télémétrie et caméravidéo, etc. Toutefois, les données actuellement disponibles ne concernent que des lahars de petit volume, responsables de dommages limités.

A l'aval des investigations, les études de vulnérabilité proposées demeurent incomplètes, car elles ne prennent pas en compte plusieurs facteurs sociaux de la vulnérabilité des populations (mobilité, origine sociale, niveau d'instruction, cohésion et stabilité des groupes sociaux, etc). De même, les facteurs culturels devraient faire l'objet d'investigations spécifiques, telles que la perception et la connaissance des phénomènes naturels et des risques par la population (D'Ercole, 1991). Cependant, il s'agit d'une recherche pluri-disciplinaire à long terme qui requiert la collaboration de géologues, géographes, sociologues, juristes, 
etc. Conduit par exemple en Californie pendant et après les crises sismiques (Mileti, ed., 1993) ou aux Philippines autour du volcan Pinatubo (Punongbayan \& Tungol, 1994), ce type de recherche n'est pas encore engagé dans la région du Merapi.

\section{Références bibliographiques}

BERTHOMMIER P., 1990. - Etude volcanologique du Merapi (CentreJava). Téphrostratigraphie et chronologie - produits éruptifs. Thèse, Université Blaise Pascal, Clermont-Fd., 223 p.

Berthommier P., Bahar I., Boudon G., Camus G., Gourgaud A., LAJOIE J. \& VINCFNI P.M., 1992. - I.e Merapi et ses éruptions : importance des mécanismes phréatomagmatiques. Bull. Soc, géol. Fr., $163,5,635-644$.

Cranimil. D.R., Boolh B., Klsumadinata K., Shimozuru D., WAI.KHR G.P.L. \& WI.STlRCAMP D., 1984. - Sourcebook for volcanic-hazard zonation. UNESCO, 97 p., Paris (see Indonesia, by Kusumadinata K., pp. 55-60).

D'FRC:OIE R., 1991. - Vulnérabilité des populations en face des risques volcaniques. Le cas de la région du Cotopaxi (Equateur). Thèse I.G.A., Université J. Fourier Grenoble I, 459 p.

GVN Bull., 1994. - Merapi 1994 cruption, Bull. Global Volcanism Network, vol. 19, 10, p. 2-3 and 11, Smithsonian Institution, Washington.

KFIFOUN K.,1994. - Evaluation des menaces en domaine volcanique actif à l'aide de la modélisation numérique. Application au volcan Merapi (Java, Indonésie). Mémoire DEA Sciences de la Terre et de l'Atmosphère, Option Terre, Université Blaise Pascal-Clermont II, 81 p. Kiallolin K., Tholri.t J.-C., Golrciaui) A., Camus G., Vincini P.M., BoURIDI:R J.-L. \& BOUION (i., 1994. - Growth and destruction of the Merapi summit dome (Central Java) : contribution of satellite imagery to hazard assessment. Intern. Volcanol. Congress, IAVCEI, Ankara (Abstract \& Poster), 6-12 September 1994.

Kiafoun K., 'Tholri:t J.-C., Lavigne F., Vinchent P.-M., Camus C. \& Gourciaul A., 1995. - Le strato-volcan Merapi (Java) : méthodes d'évaluation des menaces liées aux écoulements pyroclastiques et aux lahars. Ciéologues, sous presse.

LAVICiN: F., 1993. - Evaluation des risques associés aux lahars du Merapi, Java, Indonésie. Mémoire DEA, Géographie physique et volcanologie, Université Blaise Pascal-Clermont II, $198 \mathrm{p}$.

LAVIGiNE I. \& Thouri:T J.-C., 1994. - I es lahars du Merapi (Java) : occurrence, dynamismes et risques associés. (Résumé p.135) 15è Réunion Ann. Sciences de la Terre, Nancy, 26-28 avril 1994.

Murti D.S., editor, 1993. — The Ioma Prieta, California, Earthquake of October 17, 1989 Societal Response - Public response. U.S. Geol. Survey Prof. Paper 1553-B, 69 p. 
NEAl.I. V.E., 1976. - Lahars as major geologic hazards. Bull. Inter. Assoc. Eng. Geol. 14, 233-240.

NeWHAI.I. C.G., 1984. - Semi-quantitative assessment of changing volcanic risk at Mount St. Helens, Washington. Open-File Report 84272, U. S. Geol. Survey, 29 p.

PunUngBayan R.S. \& TUNGOI. N.M., 1994. - Impacts of the 1993 lahars and long-term lahar hazards and risks around Pinatubo volcano. Philvolcs, Manille, $40 \mathrm{p}$.

SABO Tecinicin. Clinitr, 1992. - Volcanic Hazard Map of Mount Unzen, Japan (1/25 000). Tokyo.

Simkin T., Silible'T L., M(Ci.fil.ani) L., Bridxie D., Ni:whal.i. C., I.ATITR J.H., 1981. - Volcanoes of the World. Smithsonian Institution, Hutchinson Ross Publ., 232 p.

SUMARYONO A., 1992. - Nuees ardentes deposit and its subsequent runoff through channel. Proc. Intern. Confer. Geography in the ASEAN region, 31 August-3 September 1992, Yogyakarta, $9 \mathrm{p}$.

Thoure: J.-C., 1990. - Les risques volcaniques et volcano-glaciaires dans les montagnes peuplées : identification, cartographic, évaluation. Bull. Assoc. Géogr. Fra., 2, 133-148.

THOLRL: J.-C., 1994. - Prévision des menaces et évaluation des risques volcaniques, IV, pp. 267-283, in J.-L. Bourdier, éditeur, Lee Volcanisme, Manuels et Méthodes 25, Editions BRGM, Orléans.

THOLREI J.-C. \& LAFlORCil: C., 1994. - Hazard appraisal and hazard-zone mapping of flooding and debris flowage in the Rio Combeima valley and Ibagué city, Tolima department, Colombia. Geojournal, 34-4, 407-413.

Thoure: J.-C. \& D'Ercole: R., 1995. - Vulnérabilité aux risques naturels en milicu urbain : effets, facteurs et réponses sociales. Soumis aux Cahiers des Sciences Humaines, ORSTOM, Paris.

VINCINT P. M., 1992. - Rapport de mission DRM en Indonésie (1-18 novembre 1991) : évaluation de la situation au Merapi, 34 p., inédit. V.S.I.- Paridyanto L., Riksowirogo I..D., Mitrohartono F.X.S. \& HariJJOWARSTOS S.H., 1978. - Volcanic hazard map, Merapi volcano, central Java (1/100 000). Geol. Survey of Indonesia, II, 14, Ministry of Mines, Bandung.

V.S.I.- Wirakusumah A.D., Juwarna H. \& LofBis H., 1989. Geologic map of Merapi volcano, Central Java (1/50 000). Direktorat Vulkanologi, VSI, Ministry of Mines \& Energy, Bandung.

V.S.I., VOICANOLOCiICAI. SURVI:Y OF INIOONESIA, 1990. - Gunung Merapi. $\mathrm{n}^{\circ} 122,24$ p., Dircktorat Vulcanologi, Ministry of Mines \& Energy, Bandung. 